\title{
Far di Necessità Virtù, using rare tubulopathies, Gitelman's and Bartter's syndromes, to inform the fight against COVID-19
}

\author{
Lorenzo A. Calò ${ }^{1}$ Paul A. Davis ${ }^{2}$
}

Accepted: 11 December 2020 / Published online: 2 January 2021

(C) Italian Society of Nephrology 2021

The COVID-19 pandemic has called enormous scientific attention to angiotensin converting enzyme 2 (ACE2) and other components of the renin-angiotensin system (RAS) since ACE2 plays a central role as the viral receptor initiating the SARS-CoV-2 cell infection process. In addition, ACE2 and other RAS components are known to play prominent roles in lung injury, which is a major cause of COVID19 morbidity and mortality. Our limited current understanding of SARS-CoV-2 and ACE2 roles, however, has made it difficult to delineate the pathophysiology associated with COVID-19.

The myriad of COVID-19 symptoms, alongside the prominent role of ACE2 in SARS-CoV-2 infection, has led to conflicting recommendations regarding how ACE inhibitors (ACEi) or angiotensin receptor blockers (ARBs) that target both ACE2 and other RAS-related components should be approached in COVID-19 patient care. An early concern was that increased ACE2 resulting from ACEi or ARB treatment of hypertensive patients might increase their SARSCoV-2 infection rate. This higher SARS-CoV-2 infection rate related to increased ACE2 has been criticized, while there are suggestions that administering ACEi and ARBs might be beneficial in treating COVID-19 [1]. Upregulation of ACE2 is potentially also beneficial by maintaining Ang II conversion to the vasodilatory, anti-inflammatory and antiatherosclerotic Ang 1-7 [2], while the use of ARBs could be beneficial by blocking excessive Ang II type-1 receptor

Supplementary Information The online version contains supplementary material available at https://doi.org/10.1007/s4062 0-020-00951-6.

Lorenzo A. Calò

renzcalo@unipd.it

1 Nephrology, Dialysis and Transplantation Unit, Department of Medicine, DIMED, University of Padova, Via Giustiniani 2, 35128 Padua, Italy

2 Department of Nutrition, University of California, Davis, USA
(AT1R)-mediated Ang II activation, thereby increasing Ang 1-7 levels by upregulating ACE2 activity. The potential, protective role of ACE2 in SARS-CoV-2 infection-induced COVID-19 morbidity and mortality has recently been reviewed (Supplemental Ref. [1]). In addition, not only are the effects of ACEi or ARBs potentially protective but more recently, Rho kinase (ROCK) inhibitors have also proven to be potentially useful via their ability to increase ACE2 [3].

Another line of evidence suggesting that elevated ACE2 may be of potential benefit arises from the study of renal cells as ACE2 is strongly expressed in kidney tubules. Renal impairment has been found to be the main secondary outcome of COVID-19 infection, following respiratory dysfunction (Supplemental Ref. [2]). Monteil and coworkers reported that SARS-CoV-2 directly infects human tubular kidney cells and has the ability to replicate post-infection in kidney organoids [4]. Of note, they further demonstrated that human recombinant soluble ACE2 treatment of kidney organoids significantly reduced SARS-CoV-2 infection in a dose-dependent manner [4]. These findings also argue that elevations of ACE2 are potentially beneficial.

The centrality of ACE2 and therefore of the RAS to COVID-19 provides a clear rationale for the study of systems, particularly human ones, in which ACE2 and other RAS components are altered (compared to normal). One such human system is represented by patients with Gitelman's and Bartter's syndromes (GS/BS), two rare genetic tubulopathies. We have explored and sought to better define the human RAS and RhoA/ROCK systems ([5], Supplemental Ref. [3]) by taking advantage of their endogenously activated RAS and high Ang II levels, yet blunted Ang II-mediated cardiovascular-renal effects, normotension or hypotension. Moreover, they exhibit increased and correlated levels of both ACE2 and Ang 1-7, activation of antiinflammatory, anti-apoptotic, anti-proliferative and anti-atherosclerotic defenses, reduced oxidative stress and blunted ROCK signaling [5, 6]. In addition, these patients also have up-regulated regulator of G-protein signaling (RGS)-2. 
RGS-2 acts as a negative regulator for Ang II signaling via AT1R which significantly extends to Ang II-mediated activation of the RhoA/ROCK system [5].

As COVID-19 swept through Italy earlier this year, we were presented with an opportunity to use the unique characteristics of GS/BS patients to probe for the effect of elevated ACE2 on COVID-19 in these patients. Our attention was drawn to this issue by the arguments that, in concert with the rising number of COVID-19 cases, quickly arose regarding the continuation/discontinuation of ACEi and ARB treatment in hypertensive patients contracting COVID-19. In particular, the absence of studies in humans on the effects of ACEi and ARBs on ACE2 led us to reason that this unique human model could supply much needed data. We then surveyed our cohort of GS/BS patients for the prevalence of COVID-19 with the intent to compare it to the prevalence found in the general Italian population. Our cohort consisted of $128 \mathrm{GS} / \mathrm{BS}$ patients; the relatively small number is the result of GS/BS both being rare genetic syndromes. We found no cases of COVID-19 as determined by either testing or the presence of COVID-19-related symptoms. The survey found no COVID-19 cases in our cohort, and this was statistically significant $(\mathrm{p}=0.0001 ; 95 \%$ CI $0.00-3.62 \%)$ compared with the estimated prevalence of COVID-19 in the general population of the hotspot Regions of northern Italy for COVID-19: Lombardia, Veneto and Emilia Romagna, $(8.96 \%$; $95 \%$ CI $8.96-8.99 \%)$ at the time of the survey (April 2020) [7] (manuscript submitted. The results may represent an underestimation of the prevalence of asymptomatic viral infection in our cohort since it was not possible to test every patient for SARS-CoV-2.

Accepting that we may have missed some SARS-CoV-2 infected patient(s), this occurrence would thus suggest that the effects of GS/BS renders SARS-CoV-2 infections asymptomatic and that COVID-19 symptoms are driven by ACE2 levels.

Regardless, our results indicate that GS/BS patients appear to be protected from either infection by SARS-CoV-2 itself or from the resulting clinical disease.

Our findings in GS/BS patients point to increased ACE2 as likely being beneficial, since the elevated ACE2 levels in GS/BS may be the mechanism of protection from COVID-19. However, there are other characteristics of GS/ BS patients that might beneficially affect ACE2 in a different manner. GS/BS patients have characteristic chronic metabolic alkalosis, which may have direct effects on ACE2 itself, similar to that of chloroquine/hydroxychloroquine (CQ). This potential ACE2-dependent mechanism

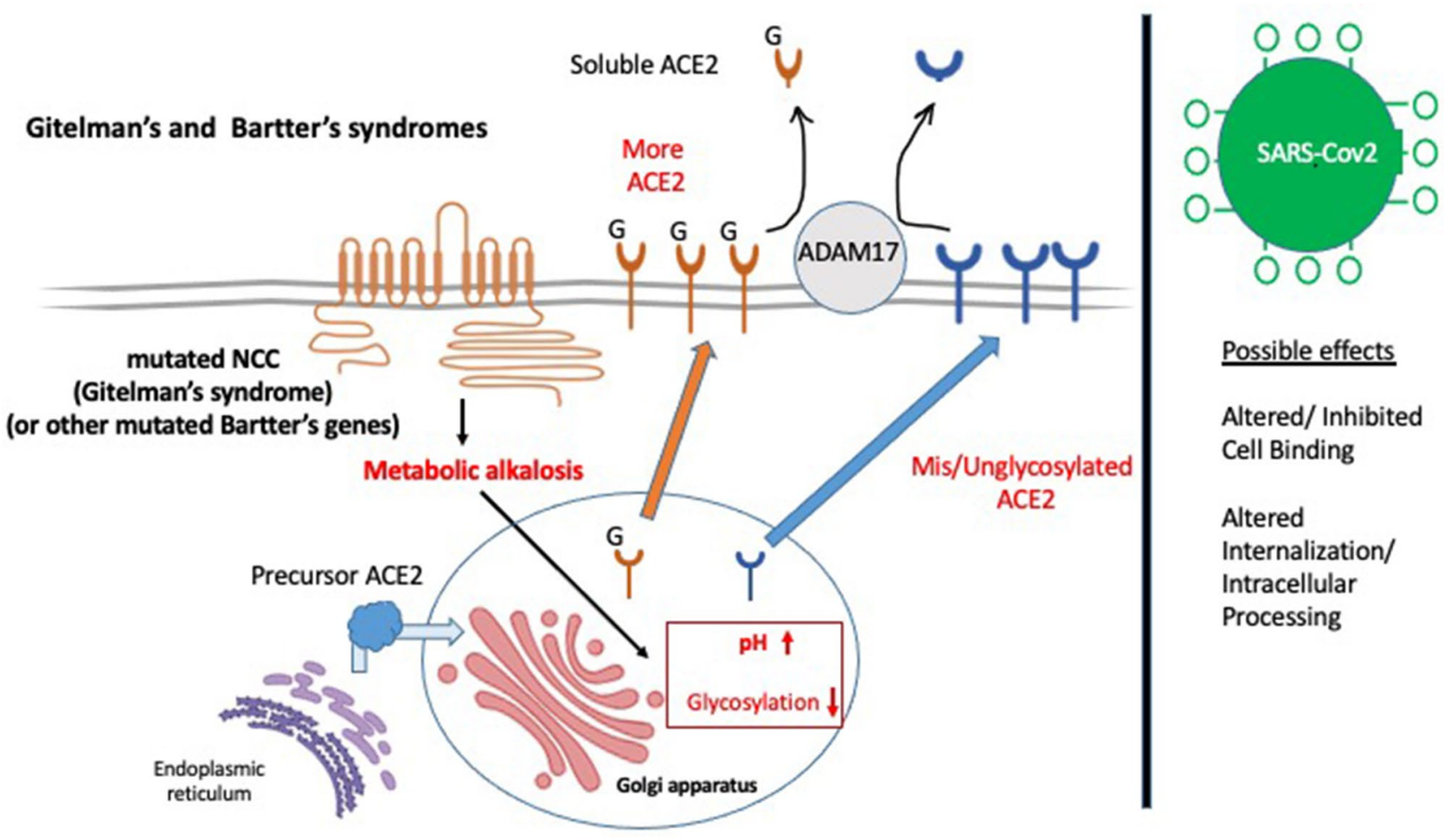

Fig. 1 Mechanism of altered ACE2 glycosylation in GS/BS 
is based on earlier reports that CQ treatment successfully treated SARS-CoV-2 infection by interfering with terminal glycosylation of ACE2 [8]. As SARS-CoV and SARSCoV-2 belong to the same viral family and share ACE2 as their means for infecting cells, this makes it likely that the negative effects of CQ on SARS-CoV ACE2 binding are shared by SARS-CoV-2. CQ inhibits infection by impairing terminal glycosylation of ACE2 by blocking the necessary acidification of Trans Golgi Network (TGN)/post-Golgi while leaving ACE2 membrane expression unaltered [8]. Thus it could be that metabolic alkalosis in GS/BS patients reproduces the same pH-dependent effect(s) on ACE2 glycosylation, thereby impacting not only the GS/BS phenotypes [Supplemental ref. 4], but in the case of COVID-19, blocking/inhibiting SARS-CoV-2 binding and the resulting COVID-19 disease ([8], Supplemental Ref. [5]) (Fig. 1). Interestingly, Brufsky suggested a different glycosylationrelated effect as being involved in the ACE2 role in SARSCoV-2 infection [9]. Based on animal experiments and on observations reported in COVID-19 patents, Brufsky suggested that hyperglycemia and diabetes lead to increased, non-enzymatically glycosylated ACE2, which concentrates in the lung. This increased glycosylated ACE2 then serves to target SARS-CoV-2 to the lung, which explains the lung as a major target for SARS-CoV-2 and why diabetes is associated with poor outcomes for COVID-19 patients. Thus, the reduction of ACE2 glycosylation resulting from enzyme inhibition in GS/BS patients that seems to be protective for COVID19 , alongside the increase in non-enzymatic glycosylation of ACE2 that has been associated with negative outcomes for COVID-19 [9] provides an impetus to further explore ACE2 glycosylation and its mechanisms linked to SARS$\mathrm{CoV}-2$. Moreover, they provide a rationale for pursuing the identification and/or synthesis of new molecules/drugs that target ACE2 glycosylation while avoiding the known potential abnormal effects of CQ on heart rhythm.

\section{Compliance with ethical standard}

Conflict of interest The authors declare no conflict of interest.

Ethical approval All procedures followed were in accordance with the ethical standards (institutional and national) and with the Helsinki Declaration of 1975, as revised in 2000.
Informed consent Informed consent was obtained from all patients.

\section{References}

1. Gurwitz D (2020) Angiotensin receptor blockers as tentative SARS-CoV-2 therapeutics. Drug Dev Res 81:537-540. https:// doi.org/10.1002/ddr.21656

2. Paz Ocaranza M, Riquelme JA, García L, Jalil JE, Chiong M, Santos RAS, Lavandero S (2020) Counter-regulatory renin-angiotensin system in cardiovascular disease. Nat Rev Cardiol 17:116-129. https://doi.org/10.1038/s41569-019-0244-8

3. Abedi F, Hayes AW, Reiter R, Karimi G (2020) Acute lung injury: the therapeutic role of Rho kinase inhibitors. Pharmacol Res. https ://doi.org/10.1016/j.phrs.2020.104736

4. Monteil V, Kwon H, Prado P, Hagelkrüys A, Wimmer RA, Stahl M, Leopoldi A, Garreta E, Del Pozo CH, Prosper F, Romero JP, Wirnsberger G, Zhang H, Slutsky AS, Conder R, Montserrat N, Mirazimi A, Penningeret JM (2020) Inhibition of SARS-CoV-2 infections in engineered human tissues using clinical-grade soluble human ACE2. Cell 181:905-913. https://doi.org/10.1016/j. cell.2020.04.004

5. Calò LA, Davis PA, Rossi GP (2014) Understanding the mechanisms of angiotensin II signaling involved in hypertension and its long-term sequelae: insights from Bartter's and Gitelman's syndromes, human models of endogenous angiotensin II signaling antagonism. J Hypertens 32:2109-2119. https://doi.org/10.1097/ HJH.0000000000000321

6. Calò LA, Schiavo S, Davis PA, Pagnin E, Mormino P, D'Angelo A, Pessina AC (2010) ACE2 and angiotensin 1-7 are increased in a human model of cardiovascular hyporeactivity: pathophysiological implications. J Nephrol 23:472-477

7. Signorelli C, Scognamiglio T, Odone A (2020) COVID-19 in Italy: impact of containment measures and prevalence estimates of infection in the general population. Acta Biomed 91:175-179. https://doi.org/10.23750/abm.v91i3-S.9511

8. Vincent MJ, Bergeron E, Benjannet S, Erickson BR, Rollin PE, Ksiazek TG, Seidah NG, Nichol ST (2005) Chloroquine is a potent inhibitor of SARS coronavirus infection and spread. Virol J 2:69. https://doi.org/10.1186/1743-422X-2-69

9. Brufsky A (2020) Hyperglycemia, hydroxychloroquine, and the COVID-19 pandemic. J Med Virol 92:770-775. https://doi. org/10.1002/jmv.25887

Publisher's Note Springer Nature remains neutral with regard to jurisdictional claims in published maps and institutional affiliations. 